\title{
Early Non-Invasive Angiographic Results of the "Button Inside” Technique for the Reattachment of Coronary Arteries in the Aortic Root Replacement
}

\author{
Carlo Canosa $^{1}$, Giuseppe Nasso ${ }^{2 *}$, Gennaro Restaino ${ }^{3}$, Carlo Maria De Filippo ${ }^{1}$, Paola Spatuzza ${ }^{1}$, \\ Nicola Testa ${ }^{1}$, Giuseppina Sallustio ${ }^{3}$, Francesco Alessandrini ${ }^{1}$ \\ ${ }^{1}$ Cardiovascular Department, Catholic University of Sacred Heart, Campobasso, Italy \\ ${ }^{2}$ Department of Cardiac Surgery, Anthea Hospital, GVM Care \& Research, Bari, Italy \\ ${ }^{3}$ Department of Radiology, Catholic University of Sacred Heart, Campobasso, Italy \\ Email: "gnasso@libero.it
}

Received February 17, 2012; revised March 29, 2012; accepted April 28, 2012

\begin{abstract}
Background and Aim: The Button technique was the operative technique generally used for the reimplantation of coronary arteries in the aortic root replacement because of reduced anastomotic complications respect to the original wrapped operation described by Bentall and De Bono. The "Button Inside" technique is a modified Button technique recently performed in all consecutive series of patients operated on aortic root replacement at our Institution. In this study we report early postoperative angiographic results of patients operated on aortic root replacement using the "Button Inside" technique. Methods: Magnetic Resonance Imaging angiography was achieved in all patients operated on aortic root replacement. Morphological characteristics of the site of coronary buttons reattachment were accurately evaluated. Results: No postoperative anastomotic leakages were detected at the site of coronary buttons anastomosis. Moreover, in this first series of postoperative control aneurisms of coronary buttons were not detected. Conclusions: Early postoperative non-invasive angiographic results of the "Button Inside" technique are encouraging. This surgical approach for the reattachment of coronary arteries is suggested in aortic root replacement when is possible to mobilize coronary arteries in order to reduce coronary artery anastomotic complications.
\end{abstract}

Keywords: Natural Asset; Financial Value; Neural Network

\section{Introduction}

The "Button Inside" technique is a modified Button technique used for the reattachment of coronary arteries in aortic root replacement (ARR) in our recent experience. Although it has been reported to be safe [1], there are no data available over the follow-up results of this technique. Herein we report the non-invasive angiographic and clinical mid-term follow-up of the earliest series of patients treated with the "Button Inside" technique.

\section{Materials and Methods}

\subsection{Patients Selection}

From July 2004 to November 2005, 8 patients underwent surgery for ARR had coronary artery reattachment using the "Button Inside" technique. Coronary buttons were mobilized and included through the openings inside the composite graft resting in the definitive position. Coro-

"Corresponding author. nary buttons were sutured from inside of the composite graft with a running 5:0 Polipropilene suture [1]. Patient data are showed in Table 1. The first patient of this series was an acute aortic dissection, one patient was a redo procedure for an ascending aorta aneurysm in previous mechanical aortic valve replacement, four patients had ascending aorta aneurysm and aortic valve incompetence, and two patients were affected by annuloaortic ectasia. None of these patients was affected by Marfan syndrome. Severe aortic incompetence was present in 7 patients. The mean age was 63.7 years (45 - 79). The local Ethical Committee approved the study protocol.

\subsection{MRI Protocol}

The Magnetic Resonance Imaging (MRI) angiography of the aortic root and of the origin of the coronary arteries was achieved with a $1.5 \mathrm{~T}$ superconducting imager (Signa; GE Medical Systems, Milwaukee, Wis) and Excite HD operating system software, along with a 8 channel cardiac phased-array coil to optimize signal detection. 
Table 1. Patient data.

\begin{tabular}{cccccc}
\hline $\begin{array}{c}\text { Patient } \\
\text { No. }\end{array}$ & Sex & $\begin{array}{c}\text { Age } \\
\text { (yrs) }\end{array}$ & $\begin{array}{c}\text { Redo } \\
(\mathrm{Y} / \mathrm{N})\end{array}$ & $\begin{array}{c}\text { Composite graft } \\
\text { (size) }\end{array}$ & $\begin{array}{c}\text { Total p.o. bleeding } \\
(\mathrm{ml})\end{array}$ \\
\hline 1 & $\mathrm{M}$ & 65 & $\mathrm{~N}$ & $25 / 28$ & 450 \\
2 & $\mathrm{~F}$ & 74 & $\mathrm{~N}$ & $23 / 26$ & 580 \\
3 & $\mathrm{M}$ & 56 & $\mathrm{~N}$ & $25 / 28$ & 420 \\
4 & $\mathrm{M}$ & 45 & $\mathrm{~N}$ & $27 / 30$ & 480 \\
5 & $\mathrm{M}$ & 72 & $\mathrm{~N}$ & $25 / 28$ & 620 \\
6 & $\mathrm{M}$ & 60 & $\mathrm{~N}$ & $25 / 28$ & 380 \\
7 & $\mathrm{M}$ & 59 & $\mathrm{Y}$ & $32^{*}$ & 450 \\
8 & $\mathrm{M}$ & 79 & $\mathrm{~N}$ & $25^{* *} / 28$ & 350 \\
\hline
\end{tabular}

p.o. $=$ Post-operative; Redo $=$ Reoperation; ${ }^{*}=$ Vascular graft; ${ }^{* *}=$ Biological aortic valve prosthesis.

The gadolinium-enhanced MR angiographic sequence was performed according to the method described by Prince [2]. Three-dimensional fast spoiled gradient-echo sequence with a spectral inversion at lipid (SPECIAL) sequence for fat suppression was used. The delay time between contrast administration and image acquisition was determined through an automated bolus detection technique (MR SmartPrep, GEMS, Waukesha, WI, USA) with the monitoring volume placed within the ascending aorta. Subtraction technique was not used in this method. Imaging parameters were as follows: 60 - $70 \mathrm{~mm}$ section thickness with 50 - 60 partitions ( $3 \mathrm{~mm}$ partition thickness), $1.5 \mathrm{~mm}$ reconstruction interval with the zero-filling interpolation technique, $4.2 \mathrm{msec}$ repetition time, 1 msec echo time, $30^{\circ}$ flip angle, $31.2 \mathrm{~Hz}$ acquisition bandwidth, $450 \times 400 \mathrm{~mm}$ field of view, and a $256 \times 192$ matrix. The section orientation was sagittal-oblique, allowing coverage of the entire thoracic aorta. Acquisition time was 16 - 20 seconds. With this pulse sequence, $\mathrm{k}$ space was acquired in the elliptical centric order, resulting in a shortened and more compact imaging period for acquisition of central k-space data. A 22 gauge plastic intravenous catheter was placed in an antecubital vein. A total of $0.1 \mathrm{mmol} / \mathrm{kg}$ of body weight of gadopentetate dimeglumine (Magnevist; Schering, Berlin, Germany) was administered at a rate of $2 \mathrm{~mL} / \mathrm{sec}$ with a power injector (Spectris, Medrad; Maastricht, The Netherlands), which was then flushed with $20 \mathrm{~mL}$ of normal saline solution, also at $2 \mathrm{~mL} / \mathrm{sec}$. Image post-processing was performed on a dedicated workstation (Advantage 4.2, General Electric Medical Systems) where source images and subvolume MIP (maximum intensity projection) reformatted images along coronal-oblique and axialoblique planes were evaluated in order to visualize the aortic root along with both origins of the coronary arteries.

\section{Results}

There were no operative or hospital deaths. No intraop- erative and postoperative myocardial ischaemia verified. No postoperative re-exploration for bleeding occurred. Total mean postoperative bleeding was $4662 \mathrm{ml}$ (350 620). All patients were submitted to postoperative follow-up. MRI angiography was performed in all patients at an average of $208.7 \pm 14$ days after surgery (range: 175 - 234 days). The MRI angiography was focused to the evaluation of morphologic characteristics of the composite graft and coronary buttons anastomoses. MRI was performed only once during the follow-up.

The average follow-up time was $4.1 \pm 0.9$ years (range: 2.5 - 6.5 years). At follow up time all patients were in good clinical conditions. No patient had postoperative sternal wound complications and/or infective complications. No ECG changes were observed and no patient was clinically suffering angina. Three patients were in NYHA I, four patients were in NYHA II and one patient was in NYHA II-III. Transthoracic two-dimensionally echocardiogramphy evaluated normal functioning aortic valve prosthesis in all patients and no perivalvular leaks prosthesis were documented. No changes between preoperative and post-operative left ventricular ejection fraction (EF) occurred. MRI was performed at the MRI angiography documented patent coronary ostia in all patients. No leakages of coronary buttons anastomoses were detected neither aneurysms of coronary buttons (Figures 1 and 2). Moreover, no leakages of proximal and distal composite graft anastomoses were detected.

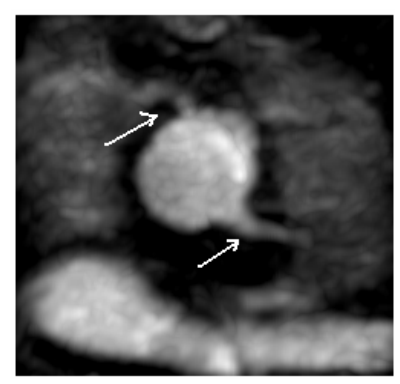

Figure 1. MRI angiography contrast-enhanced. SubvolumeMIP reconstructed view along oblique-coronal plane. Reimplanted coronary arteries (arrows) from inside of the composite graft.

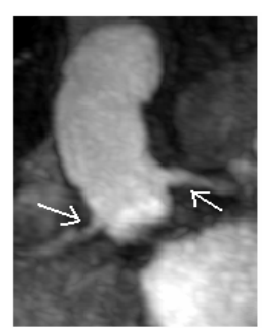

Figure 2. MRI angiography contrast-enhanced. SubvolumeMIP reconstructed view along axial-oblique plane. Reimplanted coronary arteries (arrows) from inside of the composite graft. 


\section{Comment}

The open Button technique was generally used from the beginning of 1990 as the standard surgical technique for the reattachment of coronary arteries to the composite graft in the ARR [3-6]. In selected cases of less mobilized coronary arteries Cabrol or Piehler technique may be performed to reimplant coronary arteries to the composite graft $[7,8]$. However, despite reduced complications of the Button technique respect to the in-situ coronary reimplantation of the original operation described by Bentall and De Bono [9], intraoperative and postoperative complications related to the coronary artery reimplantation may occur. Intraoperative bleeding from suture line of coronary buttons may be determinant for haemorragic complications. Adjunctive repairs may involve tearing of the coronary buttons and possible damage of the coronary ostia with consequent myocardial ischaemia. Postoperative coronay ostial complications may also occur [10-14]. Pseudoaneurysms at the site of suture line of coronary buttons, postoperative aneurysms of the surrounding aortic tissue of coronary buttons, coronary kinking, and narrowing of reimplanted coronary ostia. We recently performed the "Button inside" technique for the reattachment of coronary arteries in the aortic root replacement [1]. From July 2004 this operative technique was achieved in all consecutive patients operated on ARR at our Institution. Immediate operative results of this surgical technique for coronary artery reattachment were impressive in terms of reduced intraoperative and postoperative coronary buttons anastomotic complications. In the present study we evaluated early postoperative angiographic results of the "Button inside" technique in this first series of patients using non invasive new imaging technologies. The high performance of MRI angiography to detect postoperative complications after ARR may also increase the visualization of small pseudoaneurysms of coronary buttons non detectable by postoperative two-dimensionally echocardiography $[11,12,14]$. Moreover, MRI angiography may be reproduced several times and may represent the best non invasive choice for postoperative angiographic follow-up of patients operated on ARR expecially when postoperative leakages of the prosthetic aortic segment and coronary button anastomoses are present in asymptomatic patients treatable by urgent reoperation. In all patients of our series coronary button anastomoses were well visualized with Gadolinium-enhanced MRI imaging. No coronary ostial leakages were detected with MRI angiography. No pseudoaneurysms at the site of coronary buttons reattachment neither aneurysms of the coronary buttons have been visualized. No proximal neither distal composite graft leakages have been detected. The good clinical conditions of all patients of our series and the early postoperative MRI angiographic results are very encouraging. The "Button inside" tech- nique for coronary artery reattachment in ARR may play a key role in reduced development of intraoperative and postoperative coronary ostial complications because of coronary buttons are not under the tension of the systemic pressure free outside of the composite graft as in the open button technique. Coronary buttons are sutured under lower tension inside of the composite graft directly reiforced by the outside sutured wall around the openings of the composite graft prosthesis. Comparative and larger series of patients operated on ARR with the two different operative techniques of coronary artery reimplantation will be necessary to assess the best surgical approach for the coronary buttons reattachment in the ARR and/or aortic valve sparing.

\section{REFERENCES}

[1] C. Canosa, G. Nasso, C. M. De Filippo, et al., "The 'Button Inside' Technique for the Aortic Root Replacement: A Modified Button Technique,” Journal of Cardiac Surgery, Vol. 21, No. 4, 2006, pp. 407-409. doi:10.1111/j.1540-8191.2006.00256.x

[2] M. R. Prince, “Gadolinium-Enhanced MR Angiography,” Radiology, Vol. 191, No. 1, 1994, pp. 155-164.

[3] E. S. Crawford, L. G. Svensson, J. S. Coselli, et al., "Surgical Treatment of Aneurysm and/or Dissection of the Ascending Aorta, Transverse Aortic Arch, and Ascending Aorta and Transverse Aortic Arch,” The Journal of Thoracic and Cardiovascular Surgery, Vol. 98, No. 5, 1989, pp. 659-674.

[4] N. T. Kouchoukos, T. H. Wareing, S. F. Murphy, et al. Sixteen-Year Experience with Aortic Root Replacement. Results of 172 Operations,” Annals of Surgery, Vol. 214, No. 43, 1991, pp. 308-320. doi:10.1097/00000658-199109000-00013

[5] L. G. Svensson, E. S. Crawford, K. R. Hess, et al., "Composite Valve Graft Replacement of the Proximal Aorta: Comparison of Techniques in 348 Patients," The Annals of Thoracic Surgery, Vol. 54, No. 3, 1992, pp. 427-439. doi:10.1016/0003-4975(92)90432-4

[6] D. Pacini, F. Ranocchi, E. Angeli, et al., "Aortic Root Replacement with Composite Valve Graft," The Annals of Thoracic Surgery, Vol. 76, No. 1, 2003, pp. 90-98. doi:10.1016/S0003-4975(03)00265-0

[7] C. Cabrol, A. Pavie, I. Gangjbakhch, et al., "Complete Replacement of the Ascending Aorta with Reimplantation of the Coronary Arteries," Journal of Thoracic and Cardiovascular Surgery, Vol. 81, No. 2, 1981, pp. 309-315.

[8] M. Ito, T. Kazui, Y. Tamia, et al., "Redo Composite Valve Graft Replacement,” Journal of Cardiac Surgery, Vol. 16, No. 3, 2001, pp. 240-246. doi:10.1111/j.1540-8191.2001.tb00514.x

[9] H. Bentall and A. De Bono, "A Technique for Complete Replacement of the Ascending Aorta," Thorax, Vol. 23, No. 4, 1968, pp. 338-339. doi:10.1136/thx.23.4.338

[10] A. D. Milano, S. Pratali, G. Mecozzi, et al., "Fate of Coronary Ostial Anastomoses after the Modified Bentall Pro- 
cedure,” The Annals of Thoracic Surgery, 2003, Vol. 75, No. 6, pp. 1797-1802.

doi:10.1016/S0003-4975(03)00015-8

[11] R. Fattori, B. Descovich, P. Bertaccini, et al., "Composite Graft Replacement of the Ascending Aorta: Leakage Detection with Gadolinium-Enhanced MR Imaging," Radiology, Vol. 212, No. 2, 1999, pp. 573-577.

[12] T. G. Mesana, T. Caus, J. Y. Gaubert, et al., "Late Complications after Prosthetic Replacement of the Ascending Aorta: What Did We Learn from Routine Magnetic Resonance Imaging Follow-Up?” European Journal CardioThoracic Surgery, Vol. 18, No. 3, 2000, pp. 313-320.
doi:10.1016/S1010-7940(00)00512-1

[13] A. Panos, B. Amahzoune, J. Robin, et al., "Influence of Technique of Coronary Artery Implantation on Long-Term Results in Composite Aortic Root Replacement,” The Annals of Thoracic Surgery, Vol. 72, No. 5, 2001, pp. 14971501. doi:10.1016/S0003-4975(01)03052-1

[14] L. J. Meijboom, G. J. Nollen, N. Merchant, et al., "Frequency of Coronary Ostial Aneurysms after Aortic Root Surgery in Patients with the Marfan Syndrome,” American Journal of Cardiology, Vol. 89, No. 9, 2002, pp. 11351138. doi:10.1016/S0002-9149(02)02290-7 